\title{
EXPERIMENTS ON ENTRY OF A ROUGH CIRCULAR CYLINDER INTO WATER. APPLICATIONS TO RAINSPLASH EROSION OF SOIL
}

\author{
J. C. Wells ${ }^{1}$ and M.F. Walter ${ }^{2}$ \\ ${ }^{1}$ Member, JSCE, Ph.D., Lecturer, Faculty of Science and Engineering, Ritsumeikan University \\ (Noji Higashi 1-1-1, Kusatsu, Shiga 525-8577 Japan) \\ ${ }^{2}$ Ph.D., Professor and Chair, Dept. Agricultural \& Biological Engineering, Cornell University \\ (Riley-Robb Hall, Ithaca NY 14853, U.S.A.)
}

\begin{abstract}
Pressures generated during the initial moments of vertical entry of a circular cylinder $(10.1 \mathrm{~cm}$ radius) into a water bath at $10 \mathrm{~m} / \mathrm{s}$ were measured near the water surface by $2.5 \mathrm{~mm}$ diameter pressure transducers mounted in the sidewalls of an impact chamber, at distances of $0,1,2$, and $3 \mathrm{~cm}$ from the centerline. The incompressible theory of water entry agrees very closely with results for a smooth projectile. The theory is also found to apply to drop impact on a surface during this initial phase. Results are also presented for projectiles roughened by a fixed layer of beads. The presence of a bead layer whose thickness is $5 \%$ of the projectile radius drastically reduces the peak pressure, from 55 bar for a smooth projectile to about 20 bar. The decrease in peak pressure should not influence the amount of soil eroded during impact, which depends more critically on the extent of the region of stresses that exceed the soil's shear strength.
\end{abstract}

Key Words : Liquid-solid impact, water entry, pressure measurements, soil erosion, surface roughness

\section{INTRODUCTION}

The purpose of this work has been to experimentally probe the details of the pressure produced by the impact of a liquid drop on a (possibly rough) surface, to better understand detachment of soil by raindrop impact.

Field study ${ }^{1)}$ and experiment ${ }^{2}$ show that erosion of a soil depends on raindrop velocity. However, detailed experimental measurements of pressure produced by drop impact scem to be limited to the field of erosion of aerospace materials or turbines. At the impact velocities of these studies (on the order of $100 \mathrm{~m} / \mathrm{s}$ ), shock waves are observed to initially occur in the liquid ${ }^{3}$. These observations have led to speculation ${ }^{4)}$ that liquid compressibility might also be important in soil erosion, and correspondingly that raindrop impact pressures scale as $\rho a_{0} V$, in which $\rho$ is the water's density, $a_{0}$ is the speed of sound in water, and $V$ is the impact velocity. However, at an impact velocity on the order of $10 \mathrm{~m} / \mathrm{s}$, this phase of contact will be of negligible extent; shock waves will outrun the spreading surface of liquid-solid contact at an angle of approximately $l / M$ from the axis of symmetry, where $M$ is the impact Mach number $V / a_{0}, e . g$. at
$15 \mu \mathrm{m}$ from the axis of a $4 \mathrm{~mm}$ diameter drop.

By contrast, the incompressible theory of water entry) seems to have gone unexploited by researchers in soil erosion. Consider a circular cylinder ("projectile") of radius $R$ entering water vertically at a speed $V(t)$, as illustrated in figure 1 (b). Neglecting the change in shape of the water surface, and the curvature of the projectile, the flow is assumed to be that induced by a flat plate having a width $2 c$ which is the same as the instantaneous surface of liquid-solid contact, as sketched in figure 1(c). The complex potential in the liquid is then

$$
\phi+i \psi=V(t)\left(-z+i\left(z^{2}-c(t)^{2}\right)^{1 / 2}\right.
$$

in which the origin of the $z$-plane is the initial point of contact between projectile and water, and the $x$ axis lies along the original water surface. The halfwidth of contact $c$ will spread rapidly as the projectile enters the water, and the crux of the theory is to calculate $c(t)$ in equation 1 . Integrating the vertical velocity at the free surface over time, one predicts the rise of the free surface, and requires that the free surface meet the projectile at the contact edge. Approximating the circular cylinder by a parabola one finds 

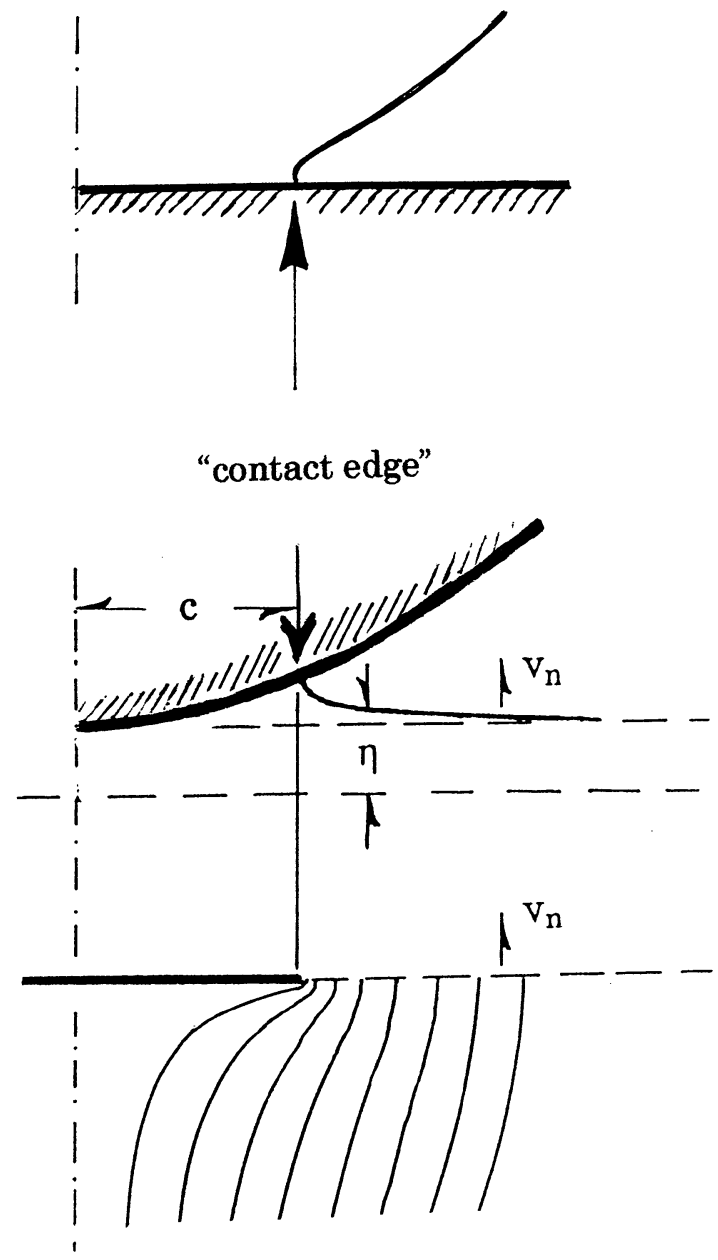

Fig. 1 (a) geometry of a liquid drop striking a solid surface (b) geometry of a circular cylinder entering water in reference frame of body. (c) Equivalent potential flow by motion of plate (ref. 5).

$$
\frac{d c}{d t}=\frac{2 R V(t)}{c}
$$

The pressure in the liquid is then

$$
\frac{-p(x ; c)}{\rho}=\frac{\partial \phi}{\partial t}+\frac{1}{2}\left|v^{2}\right|
$$

in which

$$
v=\frac{\partial \phi}{\partial x}+i \frac{\partial \phi}{\partial y}
$$

We argue that the pressures generated on a projectile during initial water entry should be nearly the same as those generated by a drop hitting a solid surface at the same impact velocity. The geometries of these two impacts are sketched in figure 1, together with the model flow of reference 5. According to the incompressible theory of liquidsolid impact outlined in equations $1-4$, the pressures on the surface are dominated by the spreading of the contact edge, $c(t)$. This spreading is essentially determined by the geometry of the gap between the solid and liquid surface, which is nearly identical for the two cases. In view of the sharp singularity in $\phi$ around $z= \pm \mathrm{c}$ (equation 1), the influence of the geometry of the free surface on pressure is primarily local, and should be effectively expressed by the angle of the free surface with respect to the solid surface. Again, at a given distance from the axis of symmetry, this angle of contact should be nearly identical for the initial stages of water entry and drop impact. In the drop impact, the free surface far from the contact edge is of course closer than for water entry, which might allow somewhat quicker release of pressures. Comparing our experimental results with numerical simulations of drop impact, however, we confirm below that the pressures are very close during the initial stage of impact studied here.

In this paper, we first compare the predictions of water entry theory (equation 3 ) with experimental pressure traces for a smooth cylindrical projectile, and find excellent agreement. We then consider results for a rough projectile, so as to model the effect of soil roughness on raindrop impact pressures. It is found that roughness simply redistributes impact pressure in time, and should little affect the amount of soil detached by a raindrop. Finally, on the basis of the excellent agreement between our experiments with the theory of incompressible liquid-solid impact, we use principles from the theory to comment on the role of ponded water in rainsplash erosion.

\section{METHOD}

Experimental impacts were produced by a springloaded "guillotine" in which a T-shaped hammer struck a projectile and drove it down into a bath of water at a nominal velocity of $10.0 \mathrm{~m} / \mathrm{s}$ (figure 2). The projectile, a $1.90 \mathrm{~cm}$ thick plate whose undersurface was an arc of radius $10.1 \mathrm{~cm}$, was sandwiched between two $1.90 \mathrm{~cm}$ thick steel sidewalls, and slid vertically between them. Two piezoelectric pressure transducers (PCB type 105A23, with built-in charge amplifiers and a sensing surface $.251 \mathrm{~cm}$ in diameter) were mounted flush with the inner surfaces of the two sidewalls, whose transverse position could be changed so as to move the transducers with respect to the vertical axis of symmetry. Pressure traces were recorded simultaneously at opposing locations of the test chamber, at distances of $x=0,1,2$, and 3 $\mathrm{cm}$ from the axis of symmetry. The water level could be controlled to within 0.25 . Before a trial, the projectile was positioned with its lowest point $2.5+/-.2 \mathrm{~cm}$ above the water surface. 
The velocity of the projectile was measured by a photocell just before it entered the water. Trials were rejected if the measured velocity differed by more than $0.4 \mathrm{~m} / \mathrm{s}$ from the nominal impact velocity of $10.0 \mathrm{~m} / \mathrm{s}$.

\section{RESULTS}

\section{(1) Smooth projectiles}

We now review our previously reported results for a smooth cylinder ${ }^{6}$. The transducer depth was set to the minimum depth which did not produce spurious peaks, and ranged from $2 \mathrm{~mm}$ below the surface at $x=0$, to $0 \mathrm{~mm}$ below the surface at $x=3 \mathrm{~cm}$ (where the water surface rose during impact to cover the transducer). Traces recorded at opposite locations were tested post-facto for symmetry according to two criteria ${ }^{6}$.

Pressure traces recorded at a given transducer position have been averaged, with individual trials shifted in time to coincide on their rising portions at a level which is half the average of the peaks of the traces to be averaged. Averaged traces are presented in figure 3, for initial water levels indicated in the caption to the figure.

To compare with the experiments, the theoretical value of pressure at a point within the liquid mass, given by equations (1) and (3) was averaged over a regular hexagonal molecule centered on the transducer position. The spread of the contact surface $c(t)$ was modelled on the basis of experimental data, and was found to agree very closely with the theoretical value (equation (2)). To calculate $V(t)$ in equation (2), projectile deceleration was modelled according to force estimates from the experimental pressure distribution. The resulting theoretical pressure traces have been overplotted on experimental traces in figure 3. Agreement between the theoretical predictions and the experimental measurements is found to be very satisfactory.

To test whether our experimental impact was an appropriate model for a drop striking a solid, the pressures on the centerline predicted by the theory of water entry have been compared with those calculated numerically by Huang et al. ${ }^{7}$ for impact of a drop on a smooth rigid surface, and found to be within $20 \%$ of each other. Note that for a drop, the increased proximity of the free surface (as compared with water entry) will most strongly affect pressures on the centerline, so this value is a conservative estimate of the difference between drop impact and water entry. On the other hand, the numerical calculations could not resolve the pressure peaks at the contact edge that are predicted by water entry theory and confirmed experimentally.
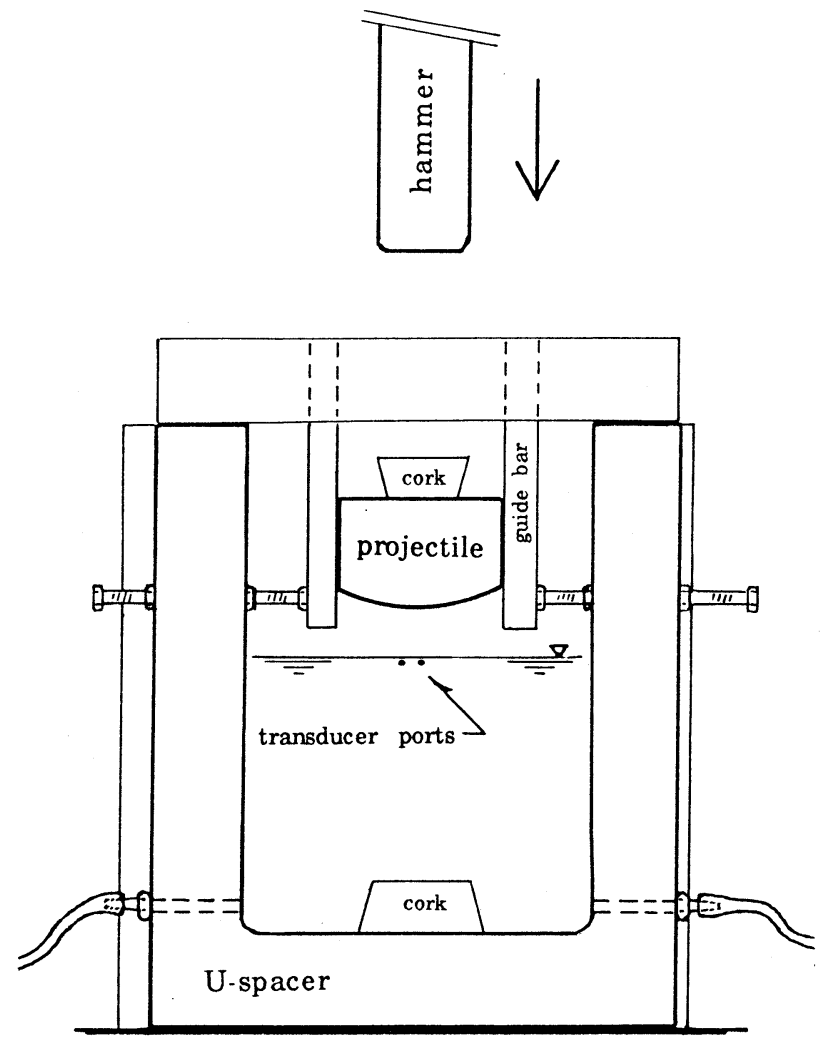

Fig. 2 Sketch of test section, viewed as with front plate removed

\section{(2) Entry of rough projectiles in water}

We now present results, unreported previously, for the peak pressure on rough projectiles using the apparatus described in the previous section. The projectiles were fabricated from plexiglass (PMMA), instead of aluminum for the smooth impacts, to allow a hexagonally close-packed monolayer of acrylic beads to be bonded strongly to the projectile with solvent (initial trials with glass beads bonded by epoxy to a metal base resulted in frequent and extensive erosion of particles.) The beads were of $3 \mathrm{~mm}$ and $5 \mathrm{~mm}$ diameter $(3 \%$ and $5 \%$ of the projectile radius). To get some idea of the effect of interstitial water, some trials were run with "wet" projectiles which were immersed in water shortly before impact, leaving a substantial fraction of the pores filled with water. Results were rather variable, seeming to depend on the time between immersion and impact, and are presented only to give a qualitative indication of the possible effect of pore water.

As with the smooth trials presented above, individual traces from a given transducer position were averaged. The threshold level at which traces were forced to overlap was chosen for the rough trials to be one quarter the average of the peaks of the individual trials. Half of the trials were run with the projectile reversed in the symmetry plane, 


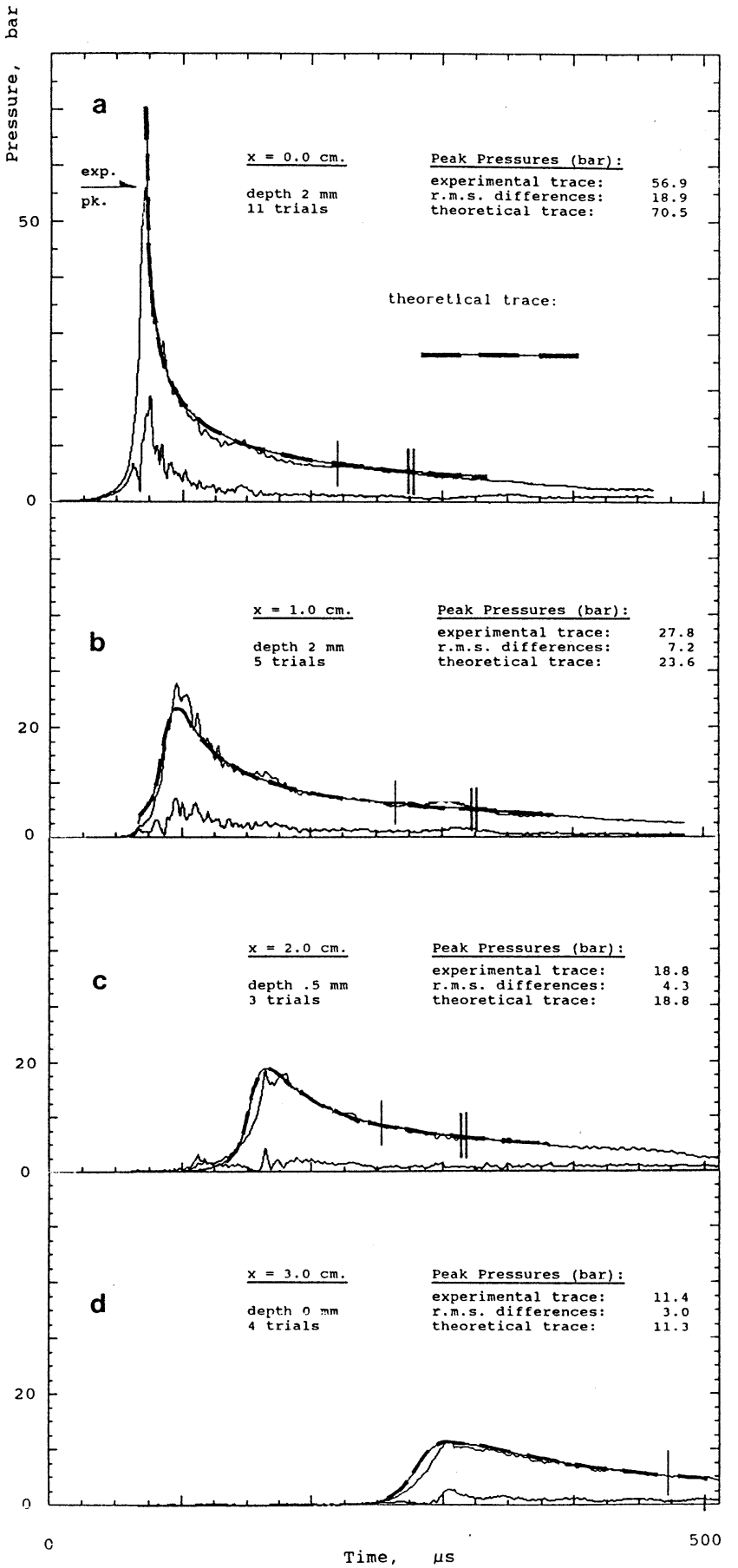

Fig. 3 Averaged pressure traces recorded at sidewalls confining impact of a cylindrical projectile. The vertical line and the following pair show when the transducer is respectively quarter and half occluded by the advancing projectile. Curve from potential theory accounts for deceleration of the projectile and changing level of the free surface.

so as to give a microscopically different alignment of the transducers with respect to the gaps in the bead layer. Since there were two pressure traces per trial, one each from the two sidewalls of the impact chamber, each averaged trace includes four microscopic geometries seen by the transducers. In fact this was not completely sufficient to smooth out local peaks resulting from geometric details of the packing of the bead layer.

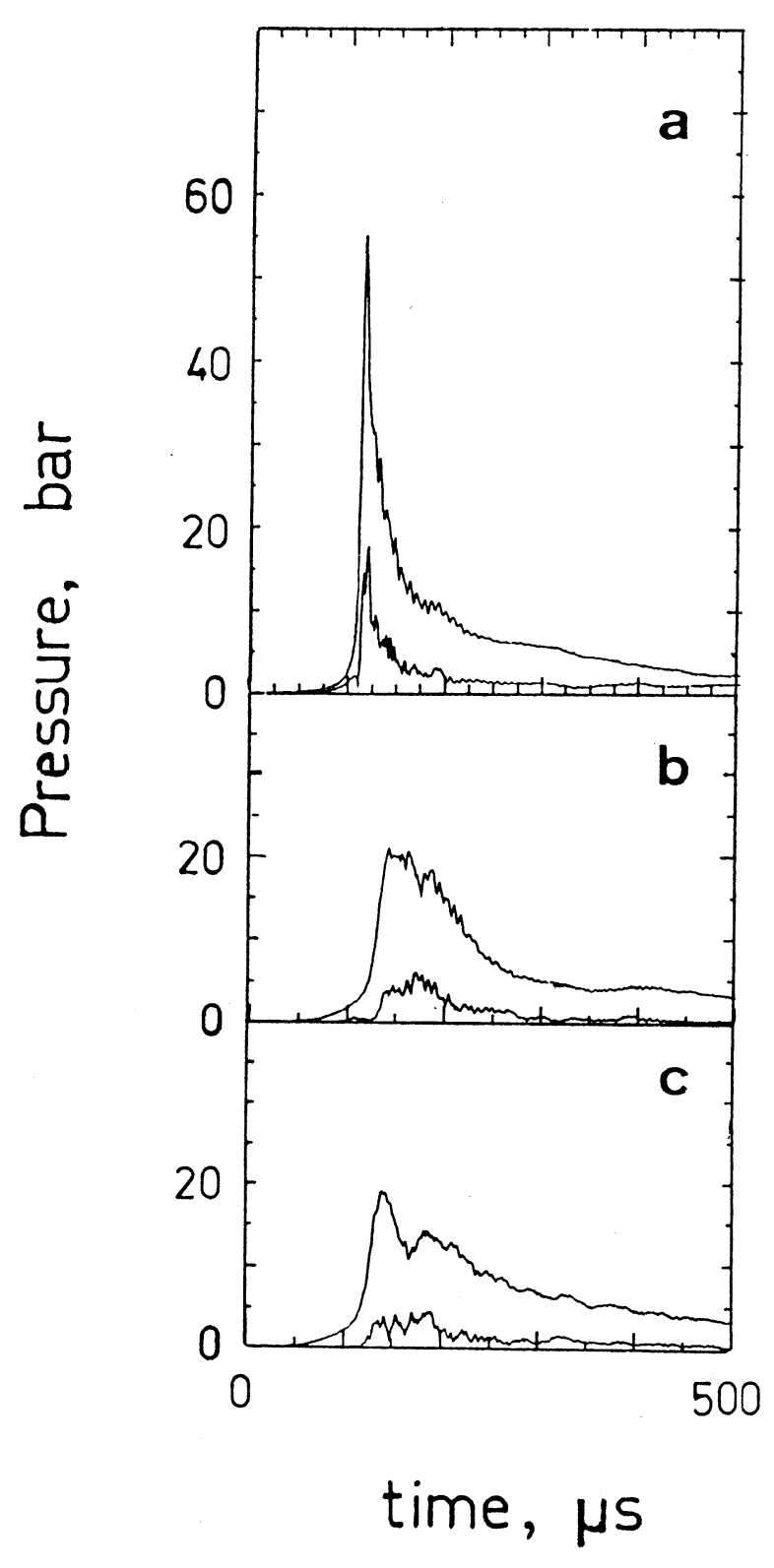

Fig. 4 Averaged pressure traces recorded on centerline in impact of a cylindrical projectile (r.m.s. traces below).

(a) smooth projectile, 11 trials, depth $2 \mathrm{~mm}$ (b) $3 \mathrm{~mm}$ beads on projectile surface, immersed in water before impact, depth $3 \mathrm{~mm}, 4$ trials; (c) same but with dry projectilel.

Figure 4 shows averaged pressure traces recorded on the centerline $(x=0)$ for the smooth projectile (a), and for the projectile roughened with $3 \mathrm{~mm}$ beads in the "wet" (b) and dry (c) condition. The curve of r.m.s. differences of the aligned individual traces from this averaged trace, also shown, indicates that the variability of the trials for the rough projectile at this location was surprisingly moderate. The most obvious effect of the rough- 


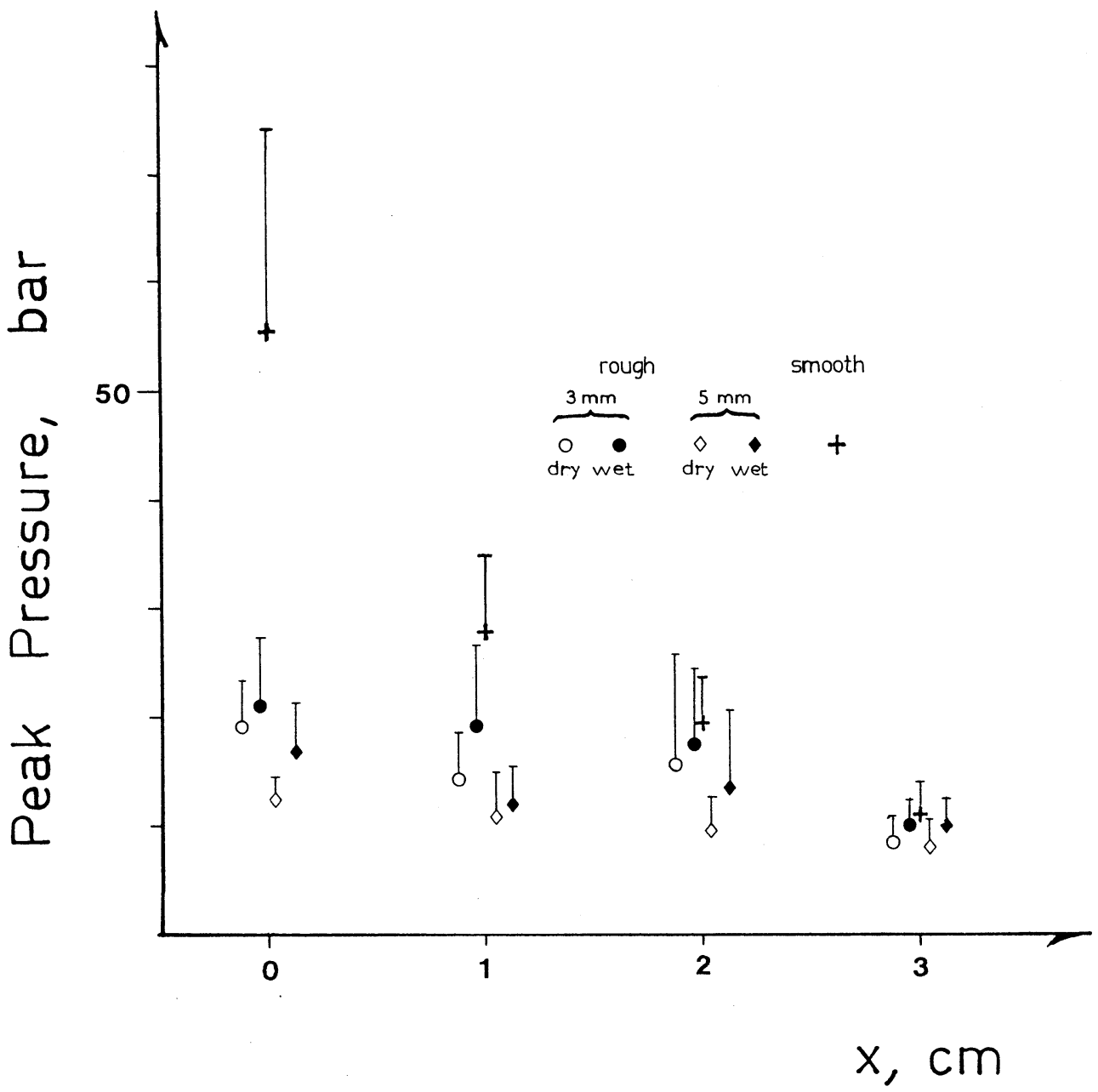

Fig. 5. Peaks of averaged traces in impact of a cylindrical projectiles (smooth, $3 \& 5 \mathrm{~mm}$ roughness, wet and dry)

ness layer is to reduce the peak pressure considerably, by a factor of nearly three, from $55 \mathrm{bar}$ to about 20 bar. However, it may be confirmed that the traces from the rough projectile are wider than from the smooth projectile, so that the total integral of pressure over time is about the same in all three cases ( $c f$. theoretical discussion below).

Figure 5 shows the peak values of the averaged pressure traces for the smooth and dry projectiles, at $x$ $=0,1,2$, and $3 \mathrm{~cm}(0,0.1,0.2,0.3$ radians) from the centerline (peaks of the traces of r.m.s. differences have been added as an indication of the uncertainty). The roughness-induced reduction in peak pressure drops off rapidly away from the axis of symmetry, so that there is nearly no difference in peak pressure at $\mathrm{x}=3 \mathrm{~cm}$. Examining the data at $\mathrm{x}=0,1$, and $2 \mathrm{~cm}$ in detail, one finds that the lower roughness $(3 \mathrm{~mm}$ beads) gives higher peaks as expected. Also, the wet projectiles give peaks which are somewhat higher than the dry case, which suggests that water in the pores of the bead layer causes water in the bath to be accelerated more rapidly upon impact.

\section{DISCUSSION}

A layer of beads has been found to reduce the maximal pressure measured on the centerline $(x=0$ $\mathrm{cm}$ ), and apparently to redistribute the pressure in time. Let us consider this effect theoretically. In equation (3), it may be verified that the second term is negligible except in an extremely narrow region at the contact edge. Then integrating with respect to time, and imposing the boundary condition on pressure at the free surface, one has

$$
\int p d t=-\rho \phi
$$

Although the boundary condition on $\phi$ for a rough boundary will be slightly different in a small region around the contact edge, i.e., there will be a narrow transition region from the condition $\frac{\partial \phi}{\partial y}=0$ inside the contact edge to the condition $\phi=0$ at the free 
surface, the effect of this difference will diminish rapidly once the contact edge has swept sufficiently far past the measuring point. We therefore expect that the value of $\phi$ will not depend on the presence of roughness and correspondingly, by equation (5), that the integral of pressure over time should be nearly independent of the roughness. In other words, the redistribution of pressure in time should be almost exact.

To summarize, the main effect of surface roughness is to redistribute the impulsive pressure in time, and this only within the fairly limited region of 0.2 radians from the axis of symmetry. It will now be argued that such a smoothing of the peaks should probably not reduce the amount of soil eroded by the impact of a raindrop. Al-Durrah \& Bradford ${ }^{8)}$ measured the shear strength of a number of soils, and showed a high correlation with the amount of soil detached by single raindrop impacts. The maximum values of shear strength they reported were about $25 \mathrm{kPa}$, or 0.25 bar. Thus, we expect that during an impact, soil will be eroded from a volume in which the shear stress exceeds this limiting value. Although we cannot convert maximal surface pressure directly to maximal shear stress in the soil, we expect that these two quantities should be of the same order of magnitude. Correspondingly, even though roughness reduced the peak centerline pressure to 10 or 20 bar from 50 bar, this reduced value should still be ample to induce failure of the soil within some volume. Thus the amount of eroded soil should not depend on the peak pressures in the region near the initial contact point, but rather on the pressures developed later during impact. This later phase will have to be considered in future work on the mechanics of soil detachment.

\section{CONCLUSION}

We have shown that the incompressible theory of water entry agrees extremely well with experimental measurements of pressure during entry of a smooth circular cylinder in water at $10 \mathrm{~m} / \mathrm{s}$, at angles from the centerline within 0.3 radians. We have also confirmed that this theory can predict, within $20 \%$, the pressures generated during the initial phase of the impact of a liquid drop on a rigid surface. Roughness is found to drastically reduce the peak pressure near the initial point of impact. However, the peak pressures generated in this region, for roughness which is $5 \%$ of the projectile's (or drop's) radius of curvature, are still an order of magnitude greater than required to detach soils.

A first conclusion, which contrasts with emphasis given by some previous workers to water compressibility ${ }^{4)}$, is thus that incompressible potential flow theory should be sufficient to predict pressures generated by a drop on a soil surface. However it will be necessary to extend the incompressible theory to later phases of impact, in which the drop geometry will have to be modelled more accurately than by the theory of water entry. To predict the amount of soil detached, it will furthermore be necessary to quantify the shear stress generated within the soil; this analysis will furthermore have to account suitably for deformation of the soil.

Since incompressible potential flow theory appears to be valid to describe drop impact, we can also conclude that a ponded layer of water must decrease peak pressures on the soil surface; by potential theory $\phi$, and $\partial \phi / \partial t$, can only have maxima on the solid surface. Experimentallyobserved increases in soil erosion due to ponded water ${ }^{9)}$ are probably due to an enlarged area of erosive action, not an increase in peak pressure.

ACKNOWLEDGEMENTS: The authors are grateful to Messrs. D. Caveney, J. Throop, and M. Jorgensen for technical assistance and advice, and to Professors J. Bartsch and A. Ruina for helpful comments during this work. Support from the Hokkaido River Disaster Prevention Research Foundation, for a research stay at the Civil Engineering Research Institute in Sapporo, is gratefully acknowledged.

\section{REFERENCES}

1) Hudson, N.W.: Proc. Trans. Rhod. Sci. Assoc. Vol. 49, pp $15-25,1961$

2) Young, R.A. and Weirsma, J.L.: The role of rainfall impact in soil detachment and transport. Water Resources Res. Vol. 9, pp. 1629-1636, 1973

3) See for example Dear, J.P. and Field, J.E.: High-speed photography of surface geometry effects in liquid-solid impact. J. Appl. Phys. Vol. 63, pp. 1015-1021, 1988

4) Tan, S.-K.: Rainfall and soil detachment. J. Hydr. Res. Vol 27, pp. 699-715, 1989

5) Wagner, $\mathrm{H}$ : Uber stoss- und gleitvorgange an der overflache von flussigkeiten. ZAMM Vol. 12, pp. 193-215. 1932

6) Wells, J.C.: Pressure measurements on a sphere and circular cylinder in incompressible water entry. submitted manuscript.

7) Huang, C., Bradford, J.M. and Cushman, J.M.: A numerical study of raindrop impact phenomena: the rigid case, Soil $S c i$ Soc. Am J. Vol. 46, pp14-19, 1982

8) Al-Durrah, M. and Bradford, J.M.: The mechanism of raindrop splash on soil surfaces. Soil Sci. Soc. Am. Vol. 46, pp. 1086-1090, 1982

9) Palmer, R.S. Water drop impact forces. Trans. ASAE 1965

(Received September 30,1998) 